Copyright (C) 201X Inderscience Enterprises Ltd. 


\title{
Distinguishability study of 3-Mass Models for Electromechanical Motion Systems
}

\section{Mathias Tantau}

Institute of Mechatronic Systems, Leibniz University Hannover, An der Universität 1, 30823, Garbsen, Germany

Tel: +49 511 762-17844 E-mail: mathias.tantau@imes.uni-hannover.de

\section{Christian Helmke}

Institute of Mechatronic Systems, Leibniz University Hannover, An der Universität 1, 30823, Garbsen, Germany

E-mail: christian.helmke@hotmail.de

\section{Lars Perner}

Lenze Automation GmbH, Am Alten Bahnhof 11, Germany

E-mail: lars.perner@lenze.com

\section{Mark Wielitzka}

Institute of Mechatronic Systems, Leibniz University Hannover, An der Universität 1, 30823, Garbsen, Germany Tel: +49 511 762-5718 E-mail: mark.wielitzka@imes.uni-hannover.de

\begin{abstract}
Physically motivated models of electromechanical motion systems are required in several applications related to control design and auto-tracking, model-based fault detection, feed-forward, and simply interpretation. However, attempts to create such models automatically via structure and parameter identification struggle with ambiguities regarding the correct internal structure of the model. Designing a reasonable set of candidate models is difficult, because it is not known which models are distinguishable and which are not. This paper gives a simple to use necessary condition for indistinguishability of multiple mass models as they are used to model the control-relevant features of motion systems. In an automated way models are generated that can be created by considering elasticities at different positions in the mechanical structures. The condition is applied to these models for the case of three masses. In three examples it is shown that the criterion simplifies the subsequent structure and parameter identification considerably by reducing the number of possible models. For higher numbers of masses, however, it would become intractable.
\end{abstract}

Keywords: indistinguishability analysis; multiple mass resonators; multiple mass models; electric drive trains; electromechanical motion systems; servo control systems; structure and parameter identification; model selection; model structure optimization; transfer function type; poles and zeros; frequency domain; frequency response function; FRF.

Reference to this paper should be made as follows: Tantau, M. et al. (2020) 'Distinguishability study of 3-mass models for electromechanical motion systems', International Journal of Modelling, Identification and Control, Vol. x, No. x, pp.xxx-xxx.

\section{Introduction}

Bright-grey box models of servo control systems have not been superseded by the currently raising blackbox models, because several applications require the knowledge of the system's internal structure. For example in the fields of control design, Kalmanfiltering, model-based fault diagnosis, and rapid control prototyping bright-grey box models are often required. Sometimes the inner structures of a given system are also useful for understanding and interpretation. 
Because modelling is time-consuming and requires expert knowledge it is seductive to automate the process of model building. Unified experiments could be conducted followed by an algorithm that identifies the parameters of several candidate models with different structures and selects the one with the best fit. In this way the structure is identified along with the parameters. Procedures of this type are called structure and parameter identification, model selection, model structure optimization and have been described in several publications: South et al. (1996); Gray et al. (1997); Aguilar et al. (2001); Madar et al. (2004); Winkler et al. (2004); dos Santos Coelho and Pessôa (2009); Tantau et al. (2019). In addition to these offline methods observers have been described that identify not only states but also model instances of switched models online (Gómez-Gutiérrez et al. (2017)).

In this study we focus on multiple mass models (MMS) as they are used to model machine tools, robots with elastic joints, stacker cranes, conveyors, etc. Identifying the best suitable MMS structure of a given system allows to determine at which point structural compliances should be considered in the model and at which point the compliance can be neglected, that is modelled by a rigid connection. The same holds for masses and rotary inertias. While the problem of selecting a suitable model complexity is excluded here we want to study the selection of the best possible multiple mass model of a certain given order.

The problem with the naive solution to identify the parameters of all candidate models and to select the one with the best fit is that of indistinguishability. The question of distinguishability of models with different structures asks whether input-output experiments exist that allow to isolate the model with the best fit or if on the contrary other candidate models with a different structure will fit just as well for any excitation. This consideration is important because otherwise the model selection algorithm would output results with treacherous certainty leading to wrong conclusions.

\subsection{Definitions}

Different notions of model distinguishability exist in the literature: Strict distinguishability deals with the question if for two models an input trajectory exists that will produce the same output, so that the models cannot be discriminated (Motchon et al. (2017)). This is not relevant for the structure and parameter identification where the question is rather if an input trajectory exists that can discriminate. Output-distinguishability and bound-distinguishability are concepts that investigate if two models can produce the same output for any input and initial state, not necessarily the same for both models (Gómez-Gutiérrez et al. (2017)). Clearly, these definitions differ from the question at hand, as explained next.

Starting point for the analysis of distinguishability is a finite set of model structures $M_{1}(\cdot), M_{2}(\cdot), \ldots$ with the same number of inputs and outputs. The parameter values $\boldsymbol{p}_{0} \in \Omega$ and $\hat{\boldsymbol{p}}_{0} \in \hat{\Omega}$ of the systems $M(\boldsymbol{p})$ and $\hat{M}(\hat{\boldsymbol{p}})$ are called equivalent if the corresponding system outputs are identical for all admissible input trajectories (Vajda (1981)). This definition is limited to single points in parameter space but can be extended to locally and structurally equivalent parameterized systems: If there are an open neighbourhood $V$ of $\boldsymbol{p}_{0}$ and $\hat{V}$ of $\hat{\boldsymbol{p}}_{0}$ where for every $p \in V$ there is an equivalent parameter value $\hat{\boldsymbol{p}} \in \hat{V}$ and vice versa, then the two systems $M(\boldsymbol{p})$ and $\hat{M}(\hat{\boldsymbol{p}})$ are called locally equivalent. Structural equivalence of the systems means that the above is true not only for a neighbourhood but for every $\boldsymbol{p} \in \Omega$, respectively $\hat{\boldsymbol{p}} \in \hat{\Omega}$, except for points of a set of zero measure (Vajda (1981)).

While the definitions of equivalence treat both models interchangeably, terms related to (in)distinguishability are sometimes used in a directed way: Model $M(\cdot)$ is said to be structurally indistinguishable (s.i.d.) from $\hat{M}(\cdot)$ if for almost any admissible $\hat{\boldsymbol{p}}$ there is at least one equivalent $\boldsymbol{p}$ (Raksanyi et al. (1985); Avdeenko and Kargin (2000)). In this case $\hat{M}(\cdot)$ is called the generating model, while $M(\cdot)$ is the model to be built (Walter et al. (1984)). If no equivalent parameter vector exists for any point in parameter space, the models are said to be structurally distinguishable (Raksanyi et al. (1985)) or structurally output distinguishable (s.o.d.) (Walter et al. (1984)). Note that $M(\cdot)$ is (s.o.d.) from $\hat{M}(\cdot)$ does not imply that $\hat{M}(\cdot)$ is (s.o.d.) from $M(\cdot)$, because $\hat{M}(\cdot)$ may be more flexible and equipped with more model parameters. Also, a model can be neither s.o.d. nor s.i.d. (Walter et al. (1984)).

As a further refinement of systems that are not structurally indistinguishable but also not structurally distinguishable Godfrey and Chapman (1989) investigated regions of indistinguishability. These are regions in $\hat{\Omega}$ in which equivalent parameter values in $\Omega$ can be found. Often, they are limited by the fact that parameter values in $\hat{\boldsymbol{p}}$ must be real and non-negative.

A different understanding of distinguishability analysis is to generically create a set of models of a certain class that are indistinguishable from a given structure or a set of structures (Godfrey and Chapman (1989)), but this usually more difficult question is not followed here.

\subsection{Methods for distinguishability analysis}

Methodologically, the investigation starts from the set of algebraic or differential equations that describe the model and depending on the method also the excitation. Then, all quantities are eliminated from the set of equations that are not necessary to describe the inputoutput behaviour, such as state variables, time, and the inputs themselves. The resulting minimal representation is called the exhaustive summary (Walter et al. (1984)) or the structural invariant vector / moment invariants (Vajda (1981)) of the model. It is then tested for the existence of solutions with the help of computer algebra and elimination theory. Examples of solvers 
for polynomial functions are given in Raksanyi et al. (1985). The minimal representation consists mostly of algebraic equations, an exception may be the local state isomorphism theorem for nonlinear state space models where differential equations are solved (Godfrey et al. (1994)).

Methods for generating the exhaustive summary include the transfer function or Laplace transform approach (Bellman and Åström (1970)) and the similarity transformation approach (Avdeenko and Kargin (2000)) for linear systems, as well as the timepower series or Taylor series approach (Pohjanpalo (1978)), generating-series approach (Raksanyi et al. (1985); Walter and Pronzato (1996)) and local state isomorphism approach (Evans et al. (2004)) for nonlinear systems. For nonlinear but rational models differential algebra has been used in Meshkat et al. (2018).

The problem of proving (in)distinguishability is closely related to identifiability. The difference is that in identifiability tests one questions the uniqueness of a solution, while in distinguishability tests one questions the existence of any solution. Accordingly, existing necessary and sufficient criteria differ significantly (Godfrey et al. (1994); Raksanyi et al. (1985)). For example, equating the Markov parameters of two models to show structural equivalence is often not readily possible because a high number of equations had to be considered, while it may be possible to show structural identifiability with only a subset of these equations.

In addition to these computationally extensive methods necessary conditions for indistinguishability have been formulated (Godfrey and Chapman (1989); Zhang et al. (1991)). They can serve to eliminate some of the candidate models in a preceding step. Recently, a web application, called DISTING has been made available, which allows to apply these conditions to linear compartmental models (Davidson et al. (2017)).

\subsection{Contributions}

Indistinguishability of different parameterized model structures is mostly known from biomedical modelling and chemical reaction kinetics (Evans et al. (2004)) represented by compartmental models (Yates et al. (2009)). In this paper the question is applied to multiple mass models as they are used to describe controlrelevant features of electromechanical motion systems. These systems allow a distinction based on the order of resonances and antiresonances. The derived criterion is often easier to apply than existing methods, even by visual comparison of the systems' frequency response functions. It is possible to exclude some or most of the candidate models in the case of two and three masses. Higher numbers of masses are not considered here, because the complexity of equations would be prohibitive.

Among the existing methods the transfer function approach is probably the most straightforward choice for models with a linear transfer function. However, it often

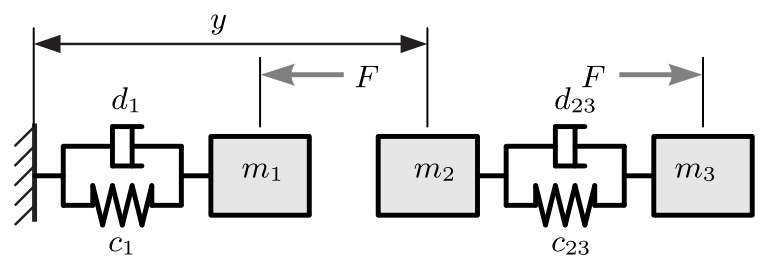

Figure 1: Exemplary multipe mass model with $N_{\mathrm{B}}=3$, $n_{\text {gap }}=1, n_{\text {act }+}=3, n_{\text {act- }}=1, n_{\text {meas }+}=2, n_{\text {meas }-}=0$

fails to provide conclusive answers for multiple mass systems as the equations tend to be long. Especially, regional distinguishability is difficult to investigate from these equations. The generic nature of all the cited methods ignores special cases that occur only for particular parameter combinations. The analysis in this papers considers also these special cases.

\section{Combinatory generation of multiple mass models}

The intention of this section is to determine how many multiple mass models can be created for a given number of masses by arranging the springs, actuator and sensor in different positions and accordingly how many different models must be investigated in the subsequent section. If the analysis shows that all the models are distinguishable, it is possible to decide based on a measured frequency response function (FRF), which model fits the measurement best, i.e. where structural compliance should be considered in the model based on a given measurement. We restrict ourselves to linear chains of masses without branching or loops and with only one actuator and one sensor, as shown in Fig. 1. $c_{i}$ and $d_{i}$ stand for linear springs and viscous dampers, respectively. Although the translational case is displayed, the analysis would work equivalently in a purely rotatory setting. Actuators and sensors are modelled as ideal transfer elements, notwithstanding the fact that more detailed models exist, including for example hysteresis (Vaiana et al. (2018)).

For a given number $N_{\mathrm{B}}$ of masses the complete set of possible models can be created by arranging the sensor, the actuator and the gap. The gap refers to the one position in the chain where the spring is missing, representing the degree of freedom of the positioner/motion system. It is located between masses $n_{\text {gap }} \in \mathbb{N}$ and $n_{\text {gap }}+1$. Actuator and sensor have one point of actuation $n_{\text {act+ }} \in \mathbb{N}$, resp. measurement $n_{\text {meas }} \in \mathbb{N}$ and a reference point against which the force is applied $n_{\text {act }-} \in \mathbb{N}$, resp. the measurement is made $n_{\text {meas }} \in \mathbb{N}$. An index of 0 refers to the rigid environment instead of a mass. The set of possible models can be constructed by varying these five indices 
under constraint of the following rules:

$$
\left\{\begin{array}{l}
0 \leq n_{\text {gap }} \leq N_{\mathrm{B}}-1, \\
0 \leq n_{\text {act }} \leq n_{\text {gap }}, \\
n_{\text {gap }}+1 \leq n_{\text {act }} \leq N_{\mathrm{B}}, \\
0 \leq n_{\text {meas }-} \leq n_{\text {gap }}, \\
n_{\text {gap }}+1 \leq n_{\text {meas }} \leq N_{\mathrm{B}}
\end{array}\right.
$$

For $N_{\mathrm{B}}=2 \ldots 5$ masses the number of possible models is given in the second row of Tab. 1.

This enumeration includes also those models where some of the masses are either not excited or the sensor signal is not sensitive to their vibration by construction. So, independent of the parameters the number of effective masses reduces and the transfer function (TF) simplifies to that of a reduced-order model. In order to exclude such degradations the following rules must be integrated in addition to (1):

$$
\left\{\begin{array}{l}
n_{\text {gap }}=0 \vee n_{\text {act }-}>0, \\
n_{\text {gap }}=0 \vee n_{\text {meas }-}>0 .
\end{array}\right.
$$

The first condition ensures excitability of all masses, while the second ensures sensitivity of the measurement to all masses. In the example in Fig. 1 the second condition is violated: Mass 1 is excited but it does not influence the measurement. As a result of including rules (2) the number of possible models reduces, see third row of Tab. 1.

Table 1 Number of possible models for different numbers of masses depending on how degradations and redundancies are counted.

\begin{tabular}{c|ccccc} 
Masses $N_{\mathrm{B}}$ & 1 & 2 & 3 & 4 & 5 \\
\hline \hline Basic combinations & 1 & 8 & 34 & 104 & 259 \\
\hline Degradation-free & 1 & 5 & 17 & 50 & 129 \\
\hline + Reduncancy-free & 1 & 3 & 11 & 29 & 73
\end{tabular}

Finally, redundant combinations must be excluded from the counting. Here, redundant refers to the situation that two models can be converted into each other by simply renaming/renumbering some of their elements. If the only difference between two models is the naming of their elements, they should not be counted as two. This can be prevented by the following additional rules:

$$
\left\{\begin{array}{l}
n_{\text {act }+} \leq n_{\text {meas }+}, \\
n_{\text {act }+} \leq n_{\text {gap }}+\left\lceil\left(N_{\mathrm{B}}-n_{\text {gap }}\right) / 2\right\rceil,
\end{array}\right.
$$

which leads to the last row of Tab. 1. The symbol \lceil\rceil means rounding up to full integers. Both rules are violated by the example in Fig. 1 .

In the next section a thorough analysis of these redundancy- and degradation-free combinations is performed in order to determine which of the models can or cannot be distinguished from an input-output measurement. We restrict the analysis to $N_{\mathrm{B}}=3$ because for $N_{\mathrm{B}}=1$ the situation is trivial and for $N_{\mathrm{B}}=2$ it is well known that both collocated models show an antiresonance-resonance (AR) behaviour independent of the parameters, while the third model has a resonance (R) FRF (Coelingh et al. (2002)). Higher counts of masses would lead to very long equations and the pursued thorough analysis of all TFs would be tedious or even intractable. For 3-mass systems the complete list of 11 possible models is shown in Tab. 2. Spring-damper elements are displayed as springs.

\section{Distinguishability criterion}

The distinguishability analysis deals with the question if a candidate model to be investigated can be parameterized in a way that it resembles a given FRF. The FRF could be obtained from a generating model or a measurement. It is uniquely defined by the gain at one frequency and the set of poles and zeros. Referring to the above definitions of indistinguishable and equivalent models or parameters, this question is clearly not answered by an analysis of structural indistinguishability, because here distinguishability is determined by characteristics that hold only in subregions of the parameter space or even in sets of zero measure, for example when poles and zeros cancel out. It is rather a question of regional indistinguishability with emphasis on special cases such as pole-zero cancellation (PZC) that occur due to dependencies among the parameters.

A necessary condition for indistinguishability in this sense is that the model to be built can show a given pattern of poles and zeros in the $\mathrm{TF}$ for physical parameters $\boldsymbol{p} \in \mathbb{R}^{+}<\infty$. Pattern means the frequency and location in the complex plane, as delineated shortly. These poles and zeros mostly show up as resonances and antiresonances in the FRF. In order to keep the analysis simple, damping is neglected in the analysis of Sec. 3 and 4 and only the inertance (force to acceleration) is considered, but the results hold also for position and velocity output and it is assumed that damping changes the FRFs, especially the frequencies of resonances and antiresonances, only minimally. As a result of these restrictions only even exponents of $s$ appear in the TF:

$$
G(s)=\frac{b_{2 n} s^{2 n}+\ldots+b_{2} s^{2}+b_{0}}{a_{2 n} s^{2 n}+\ldots+a_{2} s^{2}+a_{0}} .
$$

It is convenient to substitute $\Omega=-s^{2}$ and to discuss the location of roots in $\Omega$ rather than in $s$, because for the most common case of purely imaginary, complex conjugate roots in $s$ the equivalence in $\Omega$ is just one positive, real number.

In Fig. 2 the three possible locations of roots in the complex plane are shown. Because the coefficients are real, the roots in $\Omega$ are either real or complex conjugate. For real roots in $\Omega$, it must be further distinguished between positive and negative locations, because in the 
Table 2 Complete set of 3-mass models to be considered in the analysis. Collocated systems are marked by an asterisk $*$.

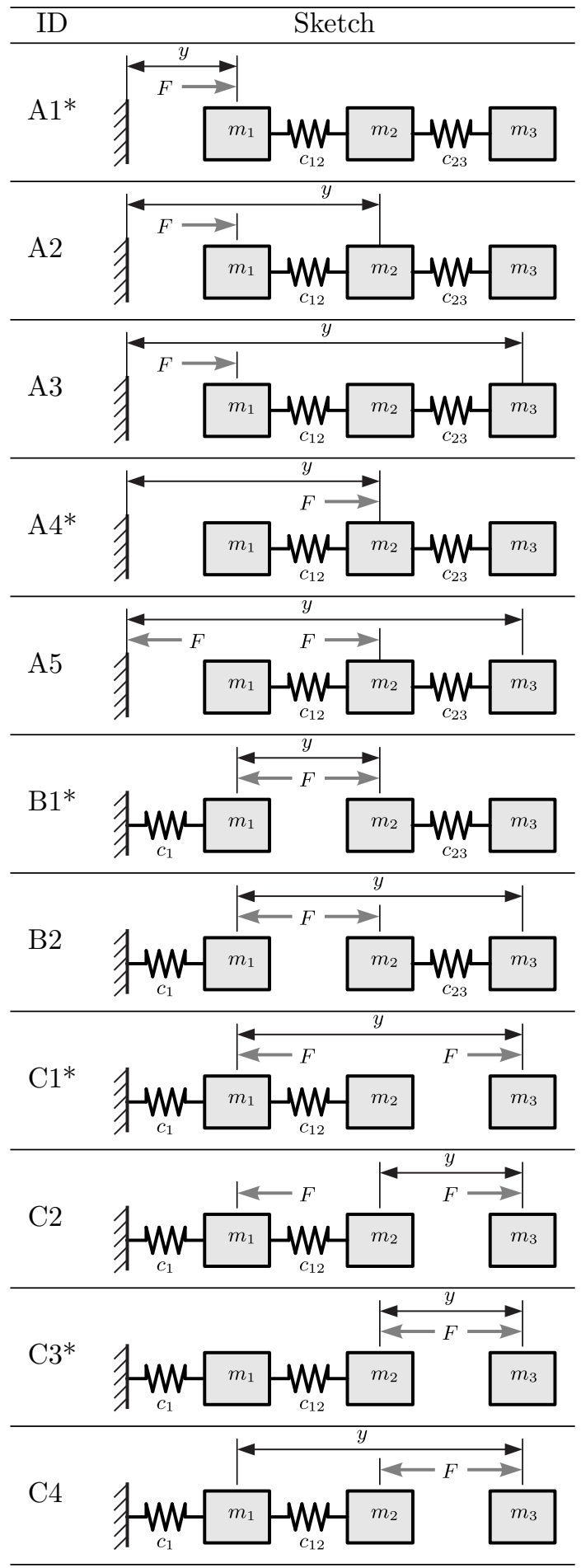

former case the roots in $s$ are complex conjugate, purely imaginary (left figure) and with damping they move into the left plane, so that the phase is changed by $\pm 180^{\circ}$. They appear as resonances and antiresonances in the FRF. In the latter case (middle figure) non-minimal phase roots $(\mathrm{nm})$ in $s$ occur and the phase remains unchanged. This is also true for the right case.
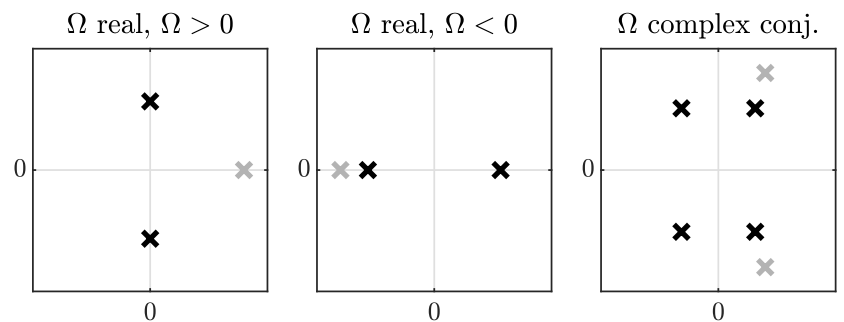

Figure 2: Locations of roots in the complex plane for $s$ (black) and $\Omega$ (grey)

Additional poles in the origin determine the type of the TF (inertance, mobility, receptance), but they are not of interest for the pattern of poles and zeros needed for the distinguishability analysis.

The pattern of poles and zeros is analysed for all eleven systems in the following section. Concatenating As for antiresonances and Rs for resonances gives the FRF type, e.g. ARR, ARAR, .... The objective is to find out which FRF types are possible for each model. Once the FRF types have been assigned, a preselection of possible models can be made for a measured or calculated FRF based on these results.

\section{Distinguishability analysis}

This section analyses the distinguishability of the eleven models shown in Tab. 2 by investigating possible FRF types as explained in Sec. 3. The analysis is started by utilising common properties of multiple mass systems in the first part of this section.

\subsection{Common properties of multiple mass systems}

The transfer function matrix from force to position can be written in matrix form:

$$
\boldsymbol{\alpha}(\omega)=\left(\boldsymbol{C}-\omega^{2} \boldsymbol{M}\right)^{-1} \quad \text { with } \quad s=i \omega
$$

with the stiffness matrix $\boldsymbol{C}$ and mass matrix $\boldsymbol{M}$. The eigenvalue problem

$$
\mathbf{0}=(\boldsymbol{C}-\Omega \boldsymbol{M}) \boldsymbol{X} \quad \text { with } \quad \Omega=\omega^{2}
$$

can be solved for the eigenvalues and eigenvectors, summarized in the matrices $\boldsymbol{\Lambda}$ and $\boldsymbol{\Psi}$, respectively. The eigenvalues in $\boldsymbol{\Lambda}$ are always real and nonnegative, because $\boldsymbol{C}$ is positive (semi-)definite and $\boldsymbol{M}$ is positive definite (Janschek (2010); Dresig and Holzweißig (2016)). They are identical to the poles of (4) and accordingly only the left case in Fig. 2 is encountered in the denominator polynomials of all systems.

The mass-normalized matrix of eigenvectors $\boldsymbol{\Phi}$ is defined as

$$
\boldsymbol{\Phi}=\boldsymbol{\Psi} \boldsymbol{M}_{r}^{1 / 2} \quad \text { width } \quad \boldsymbol{M}_{r}=\boldsymbol{\Psi}^{\mathrm{T}} \boldsymbol{M} \boldsymbol{\Psi} .
$$

$\Phi$ is used as a transformation to write the TF in modal coordinates:

$$
\boldsymbol{\alpha}(\omega)=\boldsymbol{\Phi}\left(\boldsymbol{\Lambda}-\omega^{2} \mathbf{I}\right)^{-1} \boldsymbol{\Phi}^{\mathrm{T}} .
$$


A single entry from force input at coordinate $k$ to position output at coordinate $j$ is given by the partial fraction form (Ewins (2000)):

$$
\alpha_{j k}(\omega)=\sum_{r=1}^{N} \frac{\Phi_{j r} \cdot \Phi_{k r}}{\Lambda_{r r}-\omega^{2}} .
$$

From (9) two more properties can be inferred: Firstly, the TF matrix is symmetric, which means that systems $\mathrm{C} 2$ and $\mathrm{C} 4$ have the same $\mathrm{TF}$ and are structurally equivalent. Secondly, the property of interlacing can be explained, i.e. zeros will always appear between the poles at $\omega^{2}=\Lambda_{r r}$. Because the numerator of each summand in (9) is always positive when force and measurement coincide (collocated system, $j=k$ ), the entire fraction is positive for $\omega^{2}<\Lambda_{r r}$ and negative for $\omega^{2}>\Lambda_{r r}$. Adding these fractions in the sum of (9) leads to transitions from negative to positive between adjacent resonances, which show up as antiresonances (Ewins (2000)). This is true also for systems B1, C1, C3 where sensor and actuator are applied to two masses in a differential fashion, because the describing coordinates of the system can always be chosen accordingly. Because of the gap (unconstrained system), the receptance TF $\alpha_{j k}(\omega)$ will always have the first eigenvalue at zero and accordingly there must be a zero before the first nonzero resonance frequency. This is also true for acceleration output as the poles and zeros of the undamped system are unaffected by the sensor type. Consequently, systems A1, A4, B1, C1 and C3 are known to have ARAR FRFs, unless double resonances in combination with PZC occur, which remains to be investigated.

In the following, for each of the eleven models all possible FRF types are collected that can be encountered depending on the physical parameters. It is utilized that all physical parameters are real and greater than zero but not infinite. The analysis starts with the denominators followed by the entire TFs.

\subsection{Grouping according to the denominator}

Questions that cannot be answered by the general rules for multiple mass resonators are answered in the following by looking into the system's equations. Since the denominator of the TF is independent of the sensor and actuator locations, it is possible to divide the models into three categories depending on the denominator, which is determined exclusively by the position of the gap. These categories are marked by the leading letter of the ID in Tab. 2 (A, B, C).

Again, the substitution $\Omega=-s^{2}=\omega^{2}$ is made and the denominator is solved for roots in $\Omega$. As explained above, only the left case in Fig. 2 is expectable for the poles but it remains to be investigated if double poles can occur.

The denominator of system $\mathbf{A}$ is given by

$$
\begin{aligned}
\operatorname{Den}_{\mathrm{A}}= & m_{1 \cdot 2 \cdot 3} \Omega^{2}-\left(c_{12}\left(m_{1 \cdot 3}+m_{2 \cdot 3}\right)+c_{23} m_{1}\right. \\
& \left.m_{2+3}\right) \Omega+c_{12} c_{23} m_{1+2+3} .
\end{aligned}
$$

Here and in the following the abbreviations are used:

$$
\begin{aligned}
m_{i+j} & :=\left(m_{i}+m_{j}\right), \\
m_{i \cdot j} & :=\left(m_{i} \cdot m_{j}\right) .
\end{aligned}
$$

$D e n_{\mathrm{A}}$ has two poles in $\Omega$ :

$$
\begin{aligned}
& t_{1,2}=\frac{c_{23}}{2 m_{1 \cdot 2 \cdot 3}}\left(\mp \sqrt{R_{\mathrm{A}}}+m_{1} m_{2+3}+\frac{c_{12}}{c_{23}} m_{3} m_{1+2}\right), \\
& R_{\mathrm{A}}=m_{1}^{2} m_{2+3}^{2}-\frac{m_{2} m_{1+2+3}-m_{1 \cdot 3}}{c_{23} /\left(2 c_{12} m_{1 \cdot 3}\right)}+\frac{m_{3} m_{1+2}^{2}}{c_{23}^{2} / c_{12}^{2}} .
\end{aligned}
$$

They are always distinct as can be shown by solving for the spring constants with respect to $t_{1}$ and $t_{2}$ :

$$
\begin{aligned}
& c_{12}=\frac{m_{1 \cdot 2}}{2 m_{1+2}}\left( \pm \sqrt{R_{1}}+t_{1}+t_{2}\right), \\
& c_{23}=\frac{m_{2 \cdot 3}}{2 m_{2+3}}\left(\mp \sqrt{R_{1}}+t_{1}+t_{2}\right), \\
& \text { with } R_{1}=\left(t_{1}-t_{2}\right)^{2}-\frac{4 m_{1 \cdot 3} t_{1} t_{2}}{m_{2} m_{1+2+3}} .
\end{aligned}
$$

Without numeric values it is not known which of the two solutions for $c_{12}$ and $c_{23}$ is correct, but that is not of interest here. For $c_{12}, c_{23}$ to be real it is necessary that $R_{1} \geq 0$ or equivalently $\left(t_{1}-t_{2}\right)^{2} \geq \frac{4 m_{1 \cdot 3} t_{1} t_{2}}{m_{2} m_{1+2+3}}$. So, $t_{1}$ and $t_{2}$ must be distinct.

The denominator of system $\mathbf{B}$ is given by

$$
\operatorname{Den}_{\mathrm{B}}=\left(c_{1}-m_{1} \Omega\right)\left(c_{23} m_{2+3}-m_{2 \cdot 3} \Omega\right)
$$

with the two poles

$$
t_{1}=\frac{c_{1}}{m_{1}}, \quad t_{2}=\frac{c_{23} m_{2+3}}{m_{2 \cdot 3}}
$$

corresponding to the left and the right part of the system separated by the gap. Depending on the parameters either $t_{1}$ or $t_{2}$ is larger:

$$
\frac{c_{1}}{c_{23}}>\frac{m_{1}}{m_{3}}+\frac{m_{1}}{m_{2}} \Leftrightarrow t_{1}>t_{2}
$$

and if equality holds in (16) both resonances coincide.

For system $\mathbf{C}$ the denominator is

$$
\operatorname{Den}_{\mathrm{C}}=-m_{1 \cdot 2 \cdot 3} \Omega^{2}+m_{3}\left(c_{12} m_{1+2}+m_{2} c_{1}\right) \Omega-c_{1} c_{12} m_{3}
$$

with the two poles:

$$
\begin{aligned}
& t_{1,2}=\frac{c_{12}}{2 m_{1 \cdot 2}}\left(\mp \sqrt{R_{\mathrm{C}}}+m_{1+2}+\frac{c_{1} m_{2}}{c_{12}}\right), \\
& R_{\mathrm{C}}=m_{1+2}^{2}-\frac{2 c_{1} m_{2}\left(m_{1}-m_{2}\right)}{c_{12}}+\frac{c_{1}^{2} m_{2}^{2}}{c_{12}^{2}} .
\end{aligned}
$$

Similar to system A the spring constants can be calculated from the poles:

$$
\begin{aligned}
& c_{1}=\frac{m_{1}}{2}\left( \pm \sqrt{R_{2}}+t_{1}+t_{2}\right), \\
& c_{12}=\frac{m_{1 \cdot 2}}{2 m_{1+2}}\left(\mp \sqrt{R_{2}}+t_{1}+t_{2}\right), \\
& \text { with } \quad R_{2}=\left(t_{1}-t_{2}\right)^{2}-4 \frac{m_{2}}{m_{1}} t_{1} t_{2} .
\end{aligned}
$$


For real-valued spring constants $R_{2}$ must be positive or zero and accordingly $\left(t_{1}-t_{2}\right)^{2} \geq 4 \frac{m_{2}}{m_{1}} t_{1} t_{2}$, so double poles are not possible.

What remains is the analysis of the numerators and zeros of the TFs. Where are the zeros located with respect to each other and the poles and is PZC possible?

\subsection{Analysis of the eleven 3-mass models}

For the analysis of the zeros in the TFs it is necessary to investigate each of the eleven models separately because the zeros depend on the locations of actuators and sensors as well as on the gap. As explained in Sec. 4.1, the pattern of the collocated systems A1, A4, B1, C1, and C3 is already known, except for the possibility of PZC, which remains to be investigated. Parameter sets leading to PZC are found with the help of computer algebra. If it returns no solution with valid physical parameters, it is believed that PZC does not exist.

\subsubsection{System A1}

As this is a collocated system we only need to search for PZCs. The numerator

$$
N u m_{\mathrm{A} 1}=m_{2 \cdot 3} \Omega^{2}+\left(-m_{2+3} c_{23}-m_{3} c_{12}\right) \Omega+c_{12} c_{23}
$$

has two zeros, which must be positive and interlacing as can be inferred from the property of collocation. PZC is encountered only for $c_{12}=0$ or $c_{23}=0$, which is excluded by the assumptions on the physical parameters. As a conclusion, only ARAR FRFs are possible for this system.

\subsubsection{System A2}

The numerator of this model is given by

$$
N u m_{\mathrm{A} 2}=-m_{3} c_{12} \Omega+c_{12} c_{23} \text {. }
$$

It has only one zero $n=c_{23} / m_{3}$, which is obviously real and positive. Because this model is not collocated, it is necessary to investigate permissible orders of poles and zeros. To do so, the zero is solved for $c_{23}$ and equated with (12), which can be solved for $m_{1}$ :

$$
m_{1}=-\frac{\left(\left(n-t_{2}\right) m_{2}+m_{3} n\right)\left(\left(n-t_{1}\right) m_{2}+m_{3} n\right)}{\left(n-t_{2}\right)\left(n-t_{1}\right) m_{2}+m_{3} n^{2}} .
$$

$t_{1}$ corresponds to the lower and $t_{2}$ to the higher frequency. This expression can be utilized to test the validity of different pole-zero orders starting with $n>$ $t_{2}>t_{1}$ :

$$
\begin{aligned}
& m_{1}=-\overbrace{\frac{\left(\left(n-t_{2}\right) m_{2}+m_{3} n\right)}{>0} \overbrace{\left(\left(n-t_{1}\right) m_{2}+m_{3} n\right)}^{\underbrace{\left(n-t_{2}\right)\left(n-t_{1}\right) m_{2}+m_{3} n^{2}}}}^{>0} \\
& \Rightarrow m_{1}<0,
\end{aligned}
$$

which is not possible. In the case $t_{2}>n>t_{1}$ it is less straightforward to see if a positive solution exists for $m_{1}$ :

$$
m_{1}=-\frac{\overbrace{\left(\left(n-t_{2}\right) m_{2}+m_{3} n\right)}^{<0 \vee>0} \overbrace{\left(\left(n-t_{1}\right) m_{2}+m_{3} n\right)}^{>0}}{\underbrace{\left(n-t_{2}\right)\left(n-t_{1}\right) m_{2}+m_{3} n^{2}}_{<0 \vee>0}},
$$

because two possibilities exist:

$$
\text { 1. } m_{1}=-\frac{>0}{<0} \quad \text { or } \quad 2 . m_{1}=-\frac{<0}{>0} \text {. }
$$

For the first possibility it can be followed:

$$
\begin{gathered}
\left(n-t_{2}\right) m_{2}+m_{3} n>0 \\
\Rightarrow \frac{m_{2}}{m_{3}}<-\frac{n}{n-t_{2}}, \\
\left(n-t_{2}\right)\left(n-t_{1}\right) m_{2}+m_{3} n^{2}<0 \\
\Rightarrow \frac{m_{2}}{m_{3}}>-\frac{n^{2}}{\left(n-t_{1}\right)\left(n-t_{2}\right)} .
\end{gathered}
$$

This can be rewritten into

$$
\begin{aligned}
& \frac{n^{2}}{\left(n-t_{1}\right)\left(n-t_{2}\right)}>\frac{n}{n-t_{2}} \\
& \Rightarrow t_{1}<0,
\end{aligned}
$$

which contradicts the finding that poles must be positive and accordingly the first possibility in (25) can be abandoned. With a similar reasoning it can be shown that the second possibility is always true, so $t_{2}>n>t_{1}$ is valid.

In the case $t_{2}>t_{1}>n$, also, two possibilities exist:

$$
m_{1}=-\frac{\overbrace{\left(\left(n-t_{2}\right) m_{2}+m_{3} n\right)}^{<0 \vee>0} \overbrace{\left(\left(n-t_{1}\right) m_{2}+m_{3} n\right)}^{<0 \vee>0}}{\underbrace{\left(n-t_{2}\right)\left(n-t_{1}\right) m_{2}+m_{3} n^{2}}_{>0}} .
$$

If the first brace in the enumerator is negative and the second brace is positive, it can be followed that:

$$
\begin{aligned}
& \left(n-t_{2}\right) m_{2}+m_{3} n<0 \\
& \Rightarrow \frac{m_{2}}{m_{3}}>-\frac{n}{n-t_{2}}, \\
& \left(n-t_{1}\right) m_{2}+m_{3} n>0 \\
& \Rightarrow \frac{m_{2}}{m_{3}}<-\frac{n}{n-t_{1}} \\
& \Rightarrow t_{2}>t_{1},
\end{aligned}
$$

so this case is possible, while the opposite (first brace positive and second brace negative) is not.

$\mathrm{PZC}$ is possible for the lower resonance frequency $t_{1}$ and the condition can be found by equating $t_{1}=n$ :

$$
\frac{c_{12}}{c_{23}}=\frac{m_{1}}{m_{3}} .
$$

With only one zero the second pole cannot be compensated. In summary, the model A2 can show the FRF types ARR, RAR, and due to PZC also R. 


\subsubsection{System A3}

The denominator of this model has no zeros because of the maximal distance between actuator and sensor:

$$
N u m_{\mathrm{A} 3}=c_{12} c_{23} .
$$

As already known from the analysis of the system type A poles, this model has two distinct resonances, so the FRF is of type RR.

\subsubsection{System A4}

From the numerator

$$
N u m_{\mathrm{A} 4}=\left(c_{12}-m_{1} \Omega\right)\left(c_{23}-m_{3} \Omega\right)
$$

the zeros can be obtained:

$$
n_{1}=c_{12} / m_{1}, \quad n_{2}=c_{23} / m_{3} .
$$

Obviously, they are always real and positive. With condition (32) PZC is possible with the reduced-order TF:

$$
G_{\mathrm{PZC}}(s)=\frac{-m_{3} \Omega+c_{23}}{-m_{2 \cdot 3} \Omega+m_{1+2+3} c_{23}} .
$$

For this parameter combination the zeros also coincide. Further investigations are not necessary because the model is collocated. If the condition of PZC is not met, the FRF is ARAR, otherwise the only possible FRF type is AR, because a second PCZ is not possible for $m_{1}, m_{3}>$ 0 .

\subsubsection{System A5}

The numerator of this model is close to A2:

$$
N u m_{\mathrm{A} 5}=-m_{1} c_{23} \Omega+c_{12} c_{23}
$$

and accordingly the procedure to determine the pattern of poles and zeros is similar. Firstly, it is noted that the zero of the numerator in $\Omega\left(n=c_{12} / m_{1}\right)$ is always real and positive. In order to determine the order of resonances and antiresonances the zero is solved for $c_{12}$ and equated with (11). Solving this expression for $m_{3}$ leads to

$$
m_{3}=-\frac{\left(\left(n-t_{2}\right) m_{2}+m_{1} n\right)\left(\left(n-t_{1}\right) m_{2}+m_{1} n\right)}{\left(n-t_{2}\right)\left(n-t_{1}\right) m_{2}+m_{1} n^{2}},
$$

which is the same expression as (22), except that $m_{1}$ and $m_{3}$ are exchanged. With the same reasoning as for system A2 it can be shown that the zero may be at lower frequencies than both poles, as well as between them, but not above.

PZC is possible for the lower resonance frequency $t_{1}$ if the condition (32) is met.

\subsubsection{System B1}

The numerator of this system is given by

$$
\begin{aligned}
\operatorname{Num}_{\mathrm{B} 1}= & m_{3} m_{1+2} \Omega^{2} \\
& -\left(m_{1+2+3} c_{23}+m_{3} c_{1}\right) \Omega+c_{1} c_{23}
\end{aligned}
$$

with two zeros. As this model is collocated it is clear that the roots are real and positive and that the frequencies are interlacing, so the FRF is of the form ARAR if no PZC occurs. PZC is possible for

$$
\frac{c_{1}}{c_{23}}=\frac{m_{1}}{m_{3}}+\frac{m_{1}}{m_{2}}
$$

In this case the two poles coincide with the higher antiresonance frequency. Because of the collocation property the resulting reduced-order $\mathrm{TF}$ shows $\mathrm{AR}$ behaviour with the remaining pole and zero:

$$
t_{\mathrm{red}}=\frac{m_{2+3} c_{23}}{m_{2 \cdot 3}}, \quad n_{\mathrm{red}}=\frac{m_{1} c_{23}}{m_{3} m_{1+2}} .
$$

A PZC of the remaining pole and zero is not possible for $m_{2}>0, m_{3}>0$.

\subsubsection{System B2}

The numerator

$$
N_{u m} m_{\mathrm{B} 2}=m_{2 \cdot 3} \Omega^{2}-c_{23} m_{1+2+3} \Omega+c_{1} c_{23}
$$

has two zeros:

$$
n_{1,2}=\frac{c_{23}}{2 m_{2 \cdot 3}}\left(m_{1+2+3} \mp \sqrt{m_{1+2+3}^{2}-\frac{4 m_{2 \cdot 3} c_{1}}{c_{23}}}\right) .
$$

In contrast to the other systems the zeros can be identical and also complex. They are complex for

$$
m_{1+2+3}^{2}<\frac{4 m_{2 \cdot 3} c_{1}}{c_{23}} .
$$

In this case the corresponding four zeros in $s$ are conjugate complex and located symmetric to the imaginary axis as shown in Fig. 2, third case. At their common frequency the slope of the asymptotic amplitude rises by $+80 \mathrm{~dB} / \mathrm{dec}$, while the phase remains unchanged. If condition (44) is not fulfilled, the ordinary situation of real, positive zeros is found and real, negative zeros $n_{1,2}$ are never possible, because the root in (43) is always smaller than $m_{1+2+3}$. Both sides of (44) may be equal so that the zeros coincide.

Order of poles and zeros: In the following, possible orders of poles and zeros are determined assuming for now that all four roots are distinct and real. The equations for poles and zeros (four in total) can be used to derive expressions for poles and zeros only without physical parameters, which are then used to validate orders of resonances and antiresonances. To do so, the expressions for $t_{1}$ and $t_{2}$ from (15) are solved for $c_{1}$ and $c_{23}$, respectively. Equally, from $n_{1}$ and $n_{2}$ in (43) expressions for $c_{1}$ and $c_{23}$ are derived and equated:

$$
\begin{aligned}
c_{1} & =m_{1} t_{1}=\frac{m_{1+2+3} n_{1} n_{2}}{n_{1}+n_{2}}, \\
c_{23} & =\frac{m_{2 \cdot 3} t_{2}}{m_{2+3}}=\frac{m_{2 \cdot 3}\left(n_{1}+n_{2}\right)}{m_{1+2+3}} .
\end{aligned}
$$


From these equations two expressions for $m_{1}$ can be found:

$$
m_{1}=\frac{m_{2+3} n_{1} n_{2}}{t_{1}\left(n_{1}+n_{2}\right)-n_{1} n_{2}}=\frac{\left(t_{2}-n_{1}-n_{2}\right) m_{2+3}}{-t_{2}} .
$$

Equation (47) is solved for $t_{1}$ :

$$
t_{1}=\frac{n_{1} n_{2}}{n_{1}-t_{2}+n_{2}} .
$$

This term can easily be evaluated because it contains no physical parameters but only the frequencies of interest. In the following, different assumptions about the frequencies of the poles with respect to the zeros are made and validated along this equation. In this analysis $n_{1}$ is the lower zero, $n_{2}$ refers to the higher zero.

Case $t_{1}<n_{1}$ : From (48) it can be seen that this is only possible if $t_{2}$ is also less than $n_{1}$ :

$$
\frac{t_{1}}{n_{1}}=\frac{n_{2}}{n_{1}-t_{2}+n_{2}}<1 \Rightarrow t_{2}<n_{1},
$$

vice versa $t_{1}>n_{1}$ implies $t_{2}>n_{1}$. This rules out the RARA and RAAR arrangements.

Case $t_{1}<n_{2}$ : Similar to the case $t_{1}<n_{1}$ this is only possible if $t_{2}<n_{2}$, so $t_{1}>n_{2}$ implies $t_{2}>n_{2}$. For this reason ARAR is not a valid sequence either. Remaining FRF types for distinct and real poles and zeros are AARR, ARRA, and RRAA.

PZC: Now, the assumption of distinct poles and zeros is dropped, but we still exclude the case of complex zeros. The same condition, that leads to identical poles, see (40), causes PZC of $n_{1}$ or $n_{2}$ with both poles:

$$
n_{1 / 2}=t_{1}=t_{2}=\frac{c_{23} m_{2+3}}{m_{2 \cdot 3}} .
$$

In this case the remaining zero is given by $c_{23} m_{1} / m_{2 \cdot 3}$. Depending on the parameters the FRF type is AR or RA or if in addition to (40) $m_{1}=m_{2}+m_{3}$, both zeros are identical and a complete cancellation of all poles and zeros occurs, i.e. the 3 -mass system behaves like a 1-mass system. The FRF type is called constant (C).

Double zeros: Double zeros occur if equality holds in (44). Inserting this expression in $t_{1,2}$ it can easily be shown that for $m_{1}>m_{2+3}$ the two poles have a lower frequency than the double zero. The FRF is called $\mathrm{RRA}^{2}$. For $m_{1}<m_{2+3}$ it is the other way round, $\mathrm{A}^{2} \mathrm{RR}$. On the other hand, double poles and zeros at different frequencies cannot be expected for any parameterization because the condition that leads to identical poles also causes PZC, as said before.

Complex zeros: At last the case of complex zeros in $\bar{\Omega}$ is included. Complex zeros do not show up as antiresonances in the FRF of the undamped system, see exemplary parameterization of system B2 in Fig. 3. It is now analysed when in relation to the poles this special case can be expected.

We recall the condition for complex zeros:

$$
\text { Cond }_{1}: m_{1+2+3}^{2}-\frac{4 c_{1} m_{2 \cdot 3}}{c_{23}} \stackrel{!}{<} 0
$$

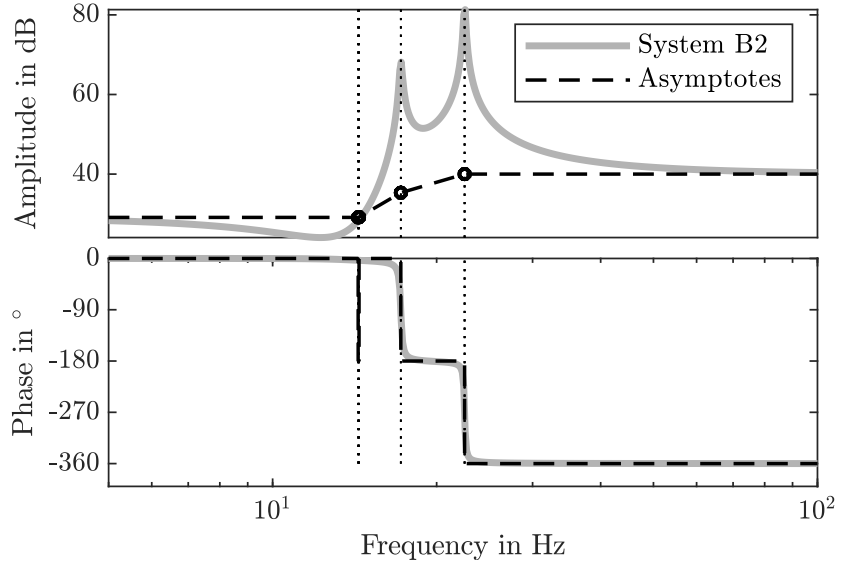

Figure 3: $\mathrm{FRF}$ of systems $\mathrm{B} 2$ with RR $(\mathrm{nm})$ characteristic. $m_{1}=0.01 \mathrm{~kg}, \quad m_{2}=0.02 \mathrm{~kg}$, $m_{3}=0.015 \mathrm{~kg}, \quad c_{1}=200 \mathrm{~N} / \mathrm{m}, \quad d_{1}=0.01 \mathrm{~N} \cdot \mathrm{s} / \mathrm{m}$, $c_{23}=100 \mathrm{~N} / \mathrm{m}, d_{23}=0.01 \mathrm{~N} \cdot \mathrm{s} / \mathrm{m}$

and the condition for identical poles:

$$
\operatorname{Cond}_{2}: \frac{c_{1}}{c_{23}} \stackrel{!}{=} \frac{m_{1}}{m_{3}}+\frac{m_{1}}{m_{2}} .
$$

In order to compare both equations they are solved for $c_{1}$ :

$$
\begin{aligned}
& \operatorname{Cond}_{1}: c_{1} \stackrel{!}{>} \frac{c_{23} m_{1+2+3}^{2}}{4 m_{2 \cdot 3}}, \\
& \operatorname{Cond}_{2}: c_{1} \stackrel{!}{=} \frac{c_{23} m_{1} m_{2+3}}{m_{2 \cdot 3}} .
\end{aligned}
$$

Equating these two expressions for $c_{1}$ leads to a contradiction:

$$
\begin{aligned}
& \frac{c_{23} m_{1} m_{2+3}}{m_{2 \cdot 3}} \stackrel{!}{>} \frac{c_{23} m_{1+2+3}^{2}}{4 m_{2 \cdot 3}} \\
& \Rightarrow\left(m_{2+3}-m_{1}\right)^{2}<0 .
\end{aligned}
$$

In other words, identical poles and complex zeros are mutually exclusive. Complex zeros can only occur if the poles are distinct, which reduces the number of FRF types to distinguish.

In summary, system B2 shows AARR, RRAA, or ARRA behaviour if the two zeros in $\Omega$ are real and distinct and the two poles in $\Omega$ are distinct. For real zeros PZC can occur once, leading to AR or RA behaviour or twice, leading to a constant gain and phase FRF (C). Under certain circumstances both zeros are identical while the poles are either both larger or smaller than the double zero $\left(\mathrm{A}^{2} \mathrm{RR}\right.$ or $\left.\mathrm{RRA}^{2}\right)$. Finally, the zeros in $\Omega$ can also become complex, which results in a non-minimal phase RR FRF with zeros that show no antiresonances but raise the slope of the amplitude response (nm).

\subsubsection{System $C 1$}

This system is collocated and it is known from Sec. 4.2 that the poles are distinct, so the only possible FRF type is ARAR. The numerator is given for completeness:

$$
\begin{aligned}
\text { Num }_{\mathrm{C} 1}= & -m_{2} m_{1+3} \Omega^{2} \\
& +\left(m_{1+2+3} c_{12}+m_{2} c_{1}\right) \Omega-c_{1} c_{12} .
\end{aligned}
$$




\subsubsection{Systems C2 and $C_{4}$}

These two systems are identical for reasons of reciprocity, with the numerator:

Num $_{\mathrm{C} 2,4}=-m_{1} m_{2} \Omega^{2}+\left(m_{1+2+3} c_{12}+m_{2} c_{1}\right) \Omega-c_{1} c_{12}$.

The zeros are given by:

$$
\begin{aligned}
& n_{1,2}=\frac{c_{12}}{2 m_{1 \cdot 2}}\left(\mp \sqrt{R_{\mathrm{C} 2,4}}+m_{1+2+3}+\frac{c_{1} m_{2}}{c_{12}}\right) \\
& R_{\mathrm{C} 2,4}=m_{1+2+3}^{2}+\frac{2 c_{1} m_{2}\left(m_{2+3}-m_{1}\right)}{c_{12}}+\frac{c_{1}^{2} m_{2}^{2}}{c_{12}{ }^{2}}
\end{aligned}
$$

It can be shown that $n_{1}$ and $n_{2}$ cannot coincide by solving the two expressions (57) for $c_{12}$ (alternatively for $\left.c_{1}\right)$ :

$$
\begin{aligned}
& c_{12}=\frac{m_{1 \cdot 2}}{2 m_{1+2+3}}\left( \pm \sqrt{R_{3}}+n_{1}+n_{2}\right), \\
& R_{3}=\left(n_{1}-n_{2}\right)^{2}-\frac{4 n_{1} n_{2} m_{2+3}}{m_{1}^{2}}
\end{aligned}
$$

For $c_{12}$ to be real it is clear that $\left(n_{1}-n_{2}\right)^{2}>\frac{4 n_{1} n_{2} m_{2+3}}{m_{1}^{2}}$. From this it also follows that $R_{\mathrm{C} 2,4}$ is never zero and because it is positive for arbitrary values, negative values can be excluded for reasons of continuity, so the zeros cannot become complex. The question if negative, real zeros exist, is equivalent to the question if $\sqrt{R_{\mathrm{C} 2,4}}$ can be greater than $m_{1+2+3}+c_{1} m_{2} / c_{12}$. As these two are already known to be positive, we can equally check the possibility of

$$
R_{\mathrm{C} 2,4}>\left(m_{1+2+3}+\frac{c_{1} m_{2}}{c_{12}}\right)^{2}
$$

Expanding these expressions and eliminating common terms leads to

$$
-2 \frac{m_{1 \cdot 2} c_{1}}{c_{12}}>2 \frac{m_{1 \cdot 2} c_{1}}{c_{12}}
$$

which is a contradiction given that all physical parameters are positive. PZC would be possible only for $c_{1}=0$ or $c_{12}=0$ or $m_{3}=0$. Inserting an arbitrary set of parameters leads to an ARRA FRF, which due to continuity and the previously mentioned properties seems to be the only possible FRF type.

\subsubsection{System C3}

The numerator is:

$$
\begin{aligned}
\operatorname{Num}_{\mathrm{C} 3}= & -m_{1} m_{2+3} \Omega^{2}+ \\
& \left(m_{1+2+3} c_{12}+c_{1} m_{2+3}\right) \Omega-c_{1} c_{12} .
\end{aligned}
$$

The zeros

$$
\begin{aligned}
& n_{1,2}=\frac{c_{12}}{2 m_{1} m_{2+3}}\left(\mp \sqrt{R_{\mathrm{C} 3}}+m_{1+2+3}+\frac{c_{1} m_{2+3}}{c_{12}}\right), \\
& R_{\mathrm{C} 3}=m_{1+2+3}^{2}-\frac{2 c_{1} m_{2+3}\left(m_{1}-m_{2+3}\right)}{c_{12}}+\frac{c_{1}^{2} m_{2+3}^{2}}{c_{12}}
\end{aligned}
$$

cannot engage in PZC, except for $c_{1}=0$ or $c_{12}=0$ or $m_{3}=0$. Accordingly, since this is a collocated system, only ARAR is possible.

\begin{tabular}{|c|c|c|}
\hline ID & Possible FRF types & Pot. similar systems \\
\hline $\mathrm{A} 1^{*}$ & ARAR & $\mathrm{A} 4, \mathrm{~B} 1, \mathrm{C} 1, \mathrm{C} 3$ \\
\hline $\mathrm{A} 2$ & ARR, RAR, R & A5 \\
\hline A3 & $\mathrm{RR}$ & - \\
\hline $\mathrm{A} 4^{*}$ & $\begin{array}{c}\text { ARAR } \\
\text { AR }\end{array}$ & $\begin{array}{c}\mathrm{A} 1, \mathrm{~B} 1, \mathrm{C} 1, \mathrm{C} 3 \\
\mathrm{~B} 1, \mathrm{~B} 2\end{array}$ \\
\hline A5 & ARR, RAR, R & $\mathrm{A} 2$ \\
\hline $\mathrm{B} 1 *$ & $\begin{array}{c}\text { ARAR } \\
\text { AR }\end{array}$ & $\begin{array}{c}\mathrm{A} 1, \mathrm{~A} 4, \mathrm{C} 1, \mathrm{C} 3 \\
\mathrm{~A} 4, \mathrm{~B} 2\end{array}$ \\
\hline B2 & $\begin{array}{c}\text { RRAA, AARR, RA } \\
\text { ARRA } \\
\text { RR }(n m) \\
\text { AR } \\
\text { A }^{2} \mathrm{RR}, \mathrm{RRA}^{2} \\
\mathrm{C}\end{array}$ & $\begin{array}{c}- \\
\mathrm{C} 2, \mathrm{C} 4 \\
- \\
\mathrm{B} 1, \mathrm{~A} 4 \\
- \\
-\end{array}$ \\
\hline $\mathrm{C} 1 *$ & ARAR & $\mathrm{A} 1, \mathrm{~A} 4, \mathrm{~B} 1, \mathrm{C} 3$ \\
\hline $\mathrm{C} 2$ & ARRA & $\mathrm{B} 2, \mathrm{C} 4$ \\
\hline $\mathrm{C} 3^{*}$ & ARAR & $\mathrm{A} 1, \mathrm{~A} 4, \mathrm{~B} 1, \mathrm{C} 1$ \\
\hline $\mathrm{C} 4$ & ARRA & $\mathrm{B} 2, \mathrm{C} 2$ \\
\hline
\end{tabular}

\subsection{Summary of the findings}

Table 3 summarizes the expectable FRF types for each model and the systems that can show the same FRF types in the right column.

Table 3 Frequency response function characteristics of the investigated systems. A: antiresonance, $\mathrm{R}$ : resonance, C: constant without resonances or antiresonances, nm: non-minimal phase, that is zeros exist in the right half of the $s$-plane

With the help of this table the procedure for identifying the model structure is as follows. Firstly, the pattern of poles and zeros is determined visually from the measured FRF. Then all systems that can show this type of FRF are chosen from the table. Finally, the parameters are identified and the results are compared. It is not guaranteed that all models that can show a particular FRF type will lead to equally good identification results, firstly because parameter bounds 


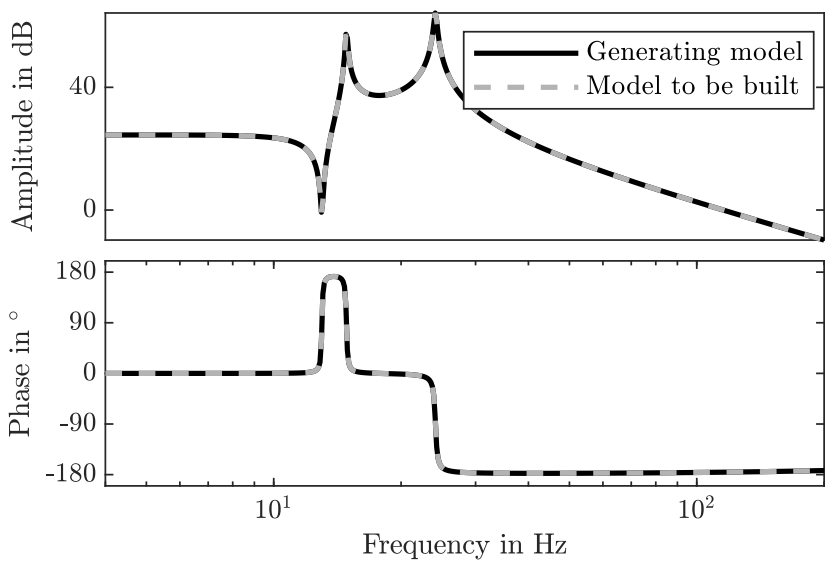

Figure 4: FRFs of systems A2 and A5 for equivalent parameter values

further restrict the flexibility of the model and secondly because the FRF type is by its nature only a necessary condition. This point is demonstrated in the examples of the next section.

\section{Examples}

This section gives some examples of situations that can occur when Tab. 3 is used to identify the model structure for a given measurement. As a first example a simulated system of type A2 is used as the generating model, parameterized according to Tab. 4 so that it shows an ARR FRF. The question is which system could have produced the FRF apart from A2, while the only prior knowledge is that all physical parameters are real, finite, and greater than zero. System A5 is the only one that can show this FRF and accordingly it is optimized to match the FRF of system A2. As can be seen in Fig. 4 both FRFs are in exact agreement. Furthermore, the parameters of system A5 are all positive, see Tab. 4. In this case it is not possible to discriminate the two systems without additional sensor information or expert knowledge.

As a side note, it can be shown that systems A2 and A5 are even structurally equivalent, because their transfer functions have the same symbolic form and all not identically constant moment invariants are independent, which is proved by the fact that the number of determinable parameters equals the number of not identically constant moment invariants, see Tab. 5 (Vajda (1981)). The number of determinable parameters represents the generic rank of the Jacobian matrix of the TF coefficients derived with respect to the parameters and the first denominator coefficient normalized to 1 (Vajda (1981)). Not identically constant are all moment invariants, except for the leading 1 in the denominator.

As a second example the system B2 is parameterized to show an ARRA behaviour and is used as the generating model. System C2 is chosen as the model to be built because its FRF type is equally ARRA.

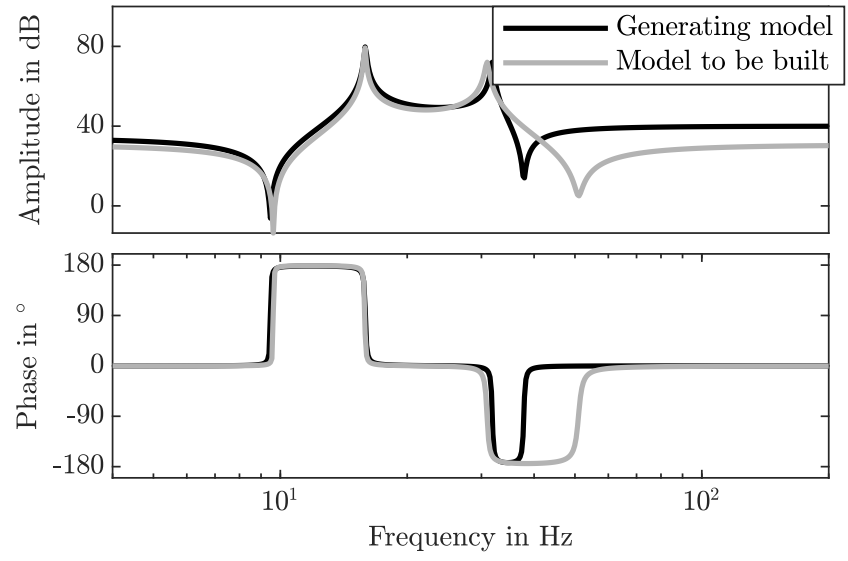

Figure 5: FRFs of systems B2 and C2 after the model to be built, $\mathrm{C} 2$, has been optimized to resemble the generating model, B2

However, it shows a different FRF after optimizing its parameters, see Fig. 5, Tab. 6. The reason is that for system $\mathrm{C} 2$ the poles and zeros are linked in the following way:

$$
\begin{aligned}
t_{1}-n_{1} & =\frac{-m_{3} c_{12}+R 4-R 5\left(m_{3}\right)}{2 m_{1 \cdot 2}}, \\
n_{2}-t_{2} & =\frac{m_{3} c_{12}+R 4-R 5\left(m_{3}\right)}{2 m_{1 \cdot 2}},
\end{aligned}
$$

where the exact terms of the abbreviations $R 4$ and $R 5$ are not of interest here. So, once the difference $t_{1}-$ $n_{1}$ is known, the difference $t_{2}-n_{2}$ is already fixed. Although the overall number of parameters of the undamped system (i.e. 5) is sufficient to adjust the 5 degrees of freedom of the system (1 gain, 2 resonances, 2 antiresonances), the system is not flexible enough to adapt to system B2. This can be explained from the number of determinable parameters as given in Tab. 5 . For system $\mathrm{C} 2$ it is only 4 out of 5 , one degree of freedom is lost.

As a third example a measured FRF is used instead of the generating model, see Fig. 6. It stems from the horizontal axis of the mast of a stacker crane. Clearly, this FRF is of ARAR type. According to Tab. 3 all collocated systems must be further considered. In a first step only one of the models is identified in physical parameters, including damping, see the grey line in Fig. 6. Then the five free coefficients of the undamped TF are calculated from the physical parameters. Finally, the physical parameters of the other collocated systems are calculated analytically from the coefficients, see Tab. 7 . Although some of the resulting parameters differ by almost one order of magnitude, it is probably difficult to decide which model is correct.

\section{Discussion}

With the findings of Sec. 4 a necessary condition for indistinguishability of models is established that allows 
Table 4 Parameters of the models A2 and A5 corresponding to the FRF in Fig. 4. All masses in kg, all stiffnesses in N/m, dampings in $\mathrm{N} \cdot \mathrm{s} / \mathrm{m}$.

\begin{tabular}{c|ccccccccc} 
ID & $m_{1}$ & $m_{2}$ & $m_{3}$ & $c_{1}$ & $c_{12}$ & $c_{23}$ & $d_{1}$ & $d_{12}$ & $d_{23}$ \\
\hline Generating model (A2) & 0.01 & 0.01 & 0.02 & - & 100 & 100 & - & 0.01 & 0.01 \\
Model to be built (A5) & 0.063 & 0.015 & 0.010 & 444 & - & 173 & 0.039 & - & 0.016
\end{tabular}

Table 5 Number of determinable parameters for the eleven 3-mass systems with 5 parameters each and number of not identically constant moment invariants. Damping is not considered.

\begin{tabular}{c|ccccccccccc} 
ID & A1 & A2 & A3 & A4 & A5 & B1* & B2 & C1 & C2 & C3* & C4 \\
\hline Determinable parameters & 5 & 4 & 3 & 5 & 4 & 5 & 4 & 5 & 4 & 5 & 4 \\
\hline $\begin{array}{c}\text { Not identically constant } \\
\text { moment invariants }\end{array}$ & 5 & 4 & 3 & 5 & 4 & 5 & 5 & 5 & 5 & 5 & 5
\end{tabular}

Table 6 Parameters of the models B2 and C2 corresponding to the transfer function in Fig. 5. All masses in kg, all stiffnesses in $\mathrm{N} / \mathrm{m}$, dampings in $\mathrm{N} \cdot \mathrm{s} / \mathrm{m}$.

\begin{tabular}{c|ccccccccc} 
ID & $m_{1}$ & $m_{2}$ & $m_{3}$ & $c_{1}$ & $c_{12}$ & $c_{23}$ & $d_{1}$ & $d_{12}$ & $d_{23}$ \\
\hline Generating model (B2) & 0.01 & 0.01 & 0.01 & 100 & - & 200 & 0.01 & - & 0.01 \\
Model to be built (C2) & 0.011 & 0.0044 & 0.030 & 190 & 96 & - & 0.01 & 0.01 & -
\end{tabular}

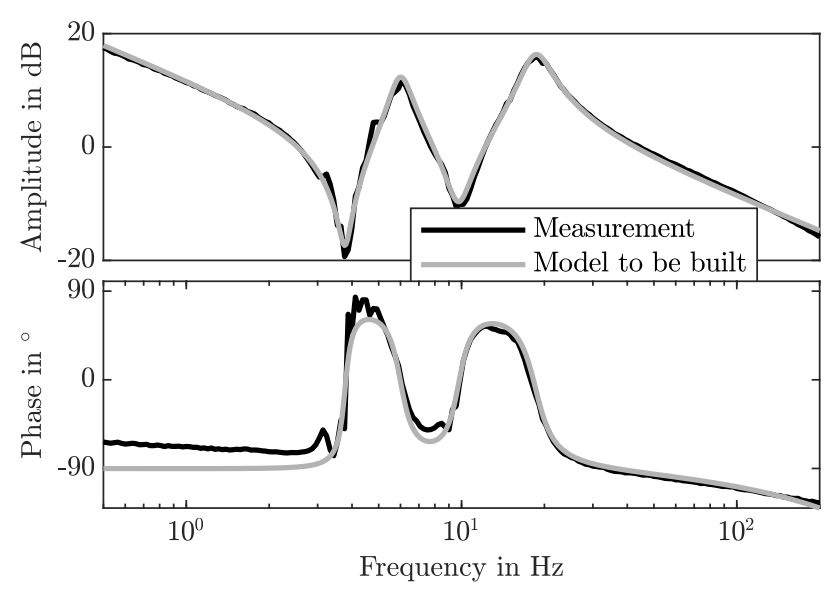

Figure 6: Experimental FRF and fitted ARAR FRF corresponding to system A1

Table 7 Parameters of the collocated models corresponding to the FRF in Fig. 6. All masses in $\mathrm{kg}$, all stiffnesses in $\mathrm{N} / \mathrm{m}$, dampings are not printed. A4 and B1 allow two solutions each being not globally identifiable.

\begin{tabular}{c|cccccc} 
ID & $m_{1}$ & $m_{2}$ & $m_{3}$ & $c_{1}$ & $c_{12}$ & $c_{23}$ \\
\hline A1 & 0.0044 & 0.0173 & 0.0184 & - & 46.8 & 14.3 \\
\hline \multirow{2}{*}{ A4 } & 0.0084 & 0.0044 & 0.0273 & - & 31.4 & 15.3 \\
& 0.0273 & 0.0044 & 0.0084 & - & 15.3 & 31.4 \\
\hline \multirow{2}{*}{ B1 } & 0.038 & 0.0049 & 0.0351 & 53.5 & - & 58.9 \\
& 0.0056 & 0.0194 & 0.0206 & 76.7 & - & 14.2 \\
\hline C1 & 0.0049 & 0.0091 & 0.040 & 31.5 & 27.4 & - \\
\hline C3 & 0.0421 & 0.0049 & 0.040 & 67.7 & 58.9 & -
\end{tabular}

to make a preselection of possible models for a given frequency response function. While the derivations are tedious and only little can be explained from general principles of machine dynamic, the results can be applied easily to a class of multi-mass resonators with up to three masses. Only in a few cases, it can be expected that it is not straightforward in practice to correctly identify the correct TF type visually, for example the non-minimal phase configuration of system B2 and the type with double zero $\left(\mathrm{A}^{2} \mathrm{RR}\right)$ of the same system. Also, with higher damping it is generally more difficult to distinguish visually between a valley and a zero.

The examples of Sec. 5 have shown that the preselection based on our necessary condition reduces the number of possible options considerably which leads to shorter calculation times of the otherwise long-lasting structure and parameter identification. A shortcoming is, however, that the rules derived here give no criterion for the final decision for one best model. In order to make this final decision further prior knowledge about parameter bounds or likely and unlikely structures would be required.

The decision if a model structure is correct or not will be exacerbated when experimental FRFs are distorted due to higher dynamics and nonlinearities. In the second example it was easy to see that system C2 was distinguishable, but in real-world experiments the model is always a simplification of reality and it will be difficult to decide if deviations are caused by these simplifications or by the wrong model structure.

Finally, the method is not arbitrarily extendible to higher numbers of masses or systems with more complex structures, e.g. branching and combined rotary and translational motion, because it cannot fully be automatized. Nevertheless, for the defined constraints of combinatorial possibilities the analysis is considered complete so that it can be utilized for up to three masses.

\section{Conclusions and Future Works}

\subsection{Conclusions}

In this paper distinguishability of multiple mass resonators was delineated as they result from considering 
elasticities and concentrated masses in electromechanical motion systems at different positions. This question is relevant for an identification of the model structure together with its parameters. The presented method allows to make a preselection of models based on the transfer function type, i.e. the order and existence of resonances, antiresonances and nonminimal phase zeros. The distinguishability analysis is easy to use and explicitly considers special parameter combinations of the generating model. This contrasts with common definitions and methods for distinguishability investigation.

It was shown that up to three masses the number of possible models is overseeable and that a thorough analysis of all options can be carried out analytically with tenable effort. Accordingly, the analysis was limited to the resulting eleven 3-mass models. Applying our necessary condition for distinguishability often cannot narrow down the set of candidates to a single model. For the final decision it is recommended to identify the parameters of the remaining candidate models and to infer the correct model from plausibility verifications of the resulting parameters.

\subsection{Future Works}

A lot more work should be done in the field of indistinguishability analysis of multiple mass systems as this is an important requirement for structure and parameter identification. While easy to use necessary conditions for equivalence of structures exist (Vajda (1984); Davidson et al. (2017)), attention should focus on sufficient conditions and the application to motion systems.

Often, the result of the analysis is that the physically correct structure cannot be determined. We, therefore, plan to investigate the benefit of additional, temporary sensors for identification and commissioning. Accelerometers and gyroscopes can be placed at different positions of the structure with little effort as a temporary solution to aid the structure and parameter identification. Moreover, instead of deciding for a single model in the selection procedure future works could focus on averaging several models with weights in proportion to their fit. An example is the work of Chen and Ding (2017).

\section{Acknowledgement}

This work was sponsored by the German Forschungsvereinigung Antriebstechnik e.V. (FVA) and the AiF Arbeitsgemeinschaft industrieller Forschungsvereinigungen "Otto von Guericke" e.V.

\section{References}

Aguilar, J., Cerrada, M., and Cordero, A. T. F. (2001). Genetic programming-based approach for system identification. Advances in Fuzzy Systems and Evolutionary Computation, Artificial Intelligence, pages 329-334.

Avdeenko, T. V. and Kargin, S. A. (2000). The problem of distinguishability of state space models. In 5th International Conference on Actual Problems of Electronic Instrument Engineering, volume 1, pages 7782. IEEE.

Bellman, R. and Åström, K. J. (1970). On structural identifiability. Mathematical biosciences, 7(3-4):329 339 .

Chen, L. and Ding, Y. (2017). Multiple model approach for nonlinear system identification with mixed-Gaussian weighting functions. International Journal of Modelling, Identification and Control, 28(4):295-306.

Coelingh, E., de Vries, T. J., and Koster, R. (2002). Assessment of mechatronic system performance at an early design stage. IEEE/ASME transactions on mechatronics, 7(3):269-279.

Davidson, N. R., Godfrey, K. R., Alquaddoomi, F., Nola, D., and DiStefano III, J. J. (2017). Disting: A web application for fast algorithmic computation of alternative indistinguishable linear compartmental models. Computer Methods and Programs in Biomedicine, 143:129-135.

dos Santos Coelho, L. and Pessôa, M. W. (2009). Nonlinear model identification of an experimental ball-andtube system using a genetic programming approach. Mechanical Systems and Signal Processing, 23(5):14341446.

Dresig, H. and Holzweißig, F. (2016). Maschinendynamik. Springer, 12th edition.

Evans, N. D., Chappell, M. J., Chapman, M. J., and Godfrey, K. R. (2004). Structural indistinguishability between uncontrolled (autonomous) nonlinear analytic systems. Automatica, 40(11):1947-1953.

Ewins, D. J. (2000). Modal testing: theory, practice, and application. Research Studies Press Ltd., 2nd edition edition.

Godfrey, K. R. and Chapman, M. J. (1989). The problem of model indistinguishability in pharmacokinetics. Journal of pharmacokinetics and biopharmaceutics, 17(2):229267.

Godfrey, K. R., Chapman, M. J., and Vajda, S. (1994). Identifiability and indistinguishability of nonlinear pharmacokinetic models. Journal of pharmacokinetics and biopharmaceutics, 22(3):229-251.

Gómez-Gutiérrez, D., Vázquez, C. R., Čelikovskỳ, S., Ramírez-Treviño, A., and Castillo-Toledo, B. (2017). On the distinguishability and observer design for singleinput single-output continuous-time switched affine systems under bounded disturbances with application to chaos-based modulation. European Journal of Control, $34: 49-58$.

Gray, G. J., Weinbrenner, T., Murray-Smith, D. J., Li, Y., and Sharman, K. C. (1997). Issues in nonlinear model structure identification using genetic programming. In Genetic Algorithms in Engineering Systems: Innovations and Applications, volume 446, pages $308-313$. 
Janschek, K. (2010). Systementwurf mechatronischer Systeme: Methoden-Modelle-Konzepte. SpringerVerlag.

Madar, J., Abonyi, J., and Szeifert, F. (2004). Genetic programming for system identification. In Intelligent Systems Design and Applications (ISDA).

Meshkat, N., Rosen, Z., Sullivant, S., et al. (2018). Algebraic tools for the analysis of state space models. In The 50th anniversary of Gröbner bases, pages 171-205. Mathematical Society of Japan.

Motchon, K. M. D., Pekpe, K. M., and Cassar, J.-P. (2017). Conditions for strict distinguishability of single-output nonlinear control-affine systems. Systems \& Control Letters, 105:20-26.

Pohjanpalo, H. (1978). System identifiability based on the power series expansion of the solution. Mathematical biosciences, 41(1-2):21-33.

Raksanyi, A., Lecourtier, Y., Walter, E., and Venot, A. (1985). Identifiability and distinguishability testing via computer algebra. Mathematical biosciences, 77(12):245-266.

South, M., Bancroft, C., Willis, M. J., and Tham, M. T. (1996). System identification via genetic programming. UKACC International Conference on Control.

Tantau, M., Perner, L., Wielitzka, M., and Ortmaier, T. (2019). Structure and parameter identification of process models with hard non-linearities for industrial drive trains by means of degenerate genetic programming. In 16th International Conference on Informatics in Control, Automation and Robotics, volume 1, pages 368-376.
Vaiana, N., Sessa, S., Marmo, F., and Rosati, L. (2018). A class of uniaxial phenomenological models for simulating hysteretic phenomena in rate-independent mechanical systems and materials. Nonlinear Dynamics, 93(3):1647-1669.

Vajda, S. (1981). Structural equivalence of linear systems and compartmental models. Mathematical Biosciences, $55(1-2): 39-64$

Vajda, S. (1984). Structural equivalence and exhaustive compartmental modeling. Mathematical biosciences, 69(1):57-75

Walter, E., Lecourtier, Y., and Happel, J. (1984). On the structural output distinguishability of parametric models, and its relations with structural identifiability. IEEE Transactions on Automatic Control, 29(1):56-57.

Walter, E. and Pronzato, L. (1996). On the identifiability and distinguishability of nonlinear parametric models. Mathematics and computers in simulation, 42(2-3):125134.

Winkler, S., Affenzeller, M., and Wagner, S. (2004). Identifying nonlinear model structures using genetic programming techniques. Citeseer.

Yates, J. W. T., Jones, R. D. O., Walker, M., and Cheung, S. Y. A. (2009). Structural identifiability and indistinguishability of compartmental models. Expert opinion on drug metabolism \& toxicology, 5(3):295-302.

Zhang, L.-Q., Collins, J. C., and King, P. H. (1991). Indistinguishability and identifiability analysis of linear compartmental models. Mathematical biosciences, 103(1):77-95. 